\title{
La technique du jodel chez les Pygmées Aka (Centrafrique). Étude phonétique et acoustique
}

\section{Susanne Fürniß}

\section{(2) OpenEdition \\ 1 Journals}

Édition électronique

URL : https://journals.openedition.org/ethnomusicologie/1588

ISSN : 2235-7688

\section{Éditeur}

ADEM - Ateliers d'ethnomusicologie

Édition imprimée

Date de publication : 1 octobre 1991

Pagination : 167-187

ISBN : 978-2-8257-0437-8

ISSN : $1662-372 X$

\section{Référence électronique}

Susanne Fürniß, « La technique du jodel chez les Pygmées Aka (Centrafrique). Étude phonétique et acoustique », Cahiers d'ethnomusicologie [En ligne], 4 | 1991, mis en ligne le 01 janvier 2012, consulté le 21 septembre 2021. URL : http://journals.openedition.org/ethnomusicologie/1588 


\title{
LA TECHNIQUE DU JODEL CHEZ LES PYGMÉES AKA (CENTRAFRIQUE). Étude phonétique et acoustique ${ }^{1}$
}

\author{
Susanne Fürniß
}

La musique des Pygmées Aka qui nomadisent dans le sud-ouest de la République Centrafricaine est essentiellement constituée de polyphonies vocales qui font appel à plusieurs modalités d'expression. Parmi celles-ci, la technique du jodel ${ }^{2}$ propre aux différents groupes de Pygmées de cette région, est un élément important dans leur chant et le distingue de celui de leurs voisins non-pygmées.

L'impression acoustique qui se dégage à l'écoute du jodel est celle d'un tissu sonore composé de plusieurs trames de timbres différents. Ceux-ci tenant une place constitutive dans la plupart des chants pygmées, nous avons été amenés à supposer que la notion de «registre», qui est liée à la différentiation des timbres, est un élément principal de cette musique. D'où notre intérêt à étudier de près la nature des timbres du jodel pygmée sous ses aspects phonétique et acoustique.

En nous basant sur l'analyse d'un corpus de musique aka, nous souhaitons apporter de nouveaux éléments à la caractérisation générale de la technique du jodel et à son utilisation dans un contexte musical. Nous nous proposons notamment de dégager les caractéristiques des registres vocaux constituant le jodel, le rôle spécifique des voyelles utilisées, et les modalités du traitement des intervalles conjoints dans des mélodies jodlées de la musique aka. Pour y parvenir, nous avons recours à l'analyse acoustique des timbres vocaux par sonagraphe et à l'étude phonétique et statistique des voyelles, pour aboutir à une analyse mélodique de l'intégration des intervalles conjoints dans le jodel aka.

Nous allons d'abord examiner les recherches antérieures sur le jodel sous ses différents aspects - musicologique, phonétique, linguistique et acoustique - pour les confronter ensuite à nos propres analyses.

Cet article est le résumé d'un mémoire de DEA que j'ai soutenu à l'Université de Paris III en 1988 et dont une traduction intégrale en allemand est actuellement sous presse. Je tiens ici à remercier vivement Simha Arom d'avoir encouragé et suivi ma recherche et d'avoir bien voulu mettre à ma disposition ses enregistrements sonores personnels qui sont à la base même de ce travail, ainsi que ses informations ethnologiques et musicologiques sans lesquelles une partie importante de cette étude n'aurait pas pu être menée à bien. Je le remercie également - ainsi que Sophie Pelletier - pour ses conseils judicieux lors de la révision du présent texte.

2 En l'absence d'un terme technique français, nous utilisons ce terme germanique avec son orthographe original.. 


\section{État de la question}

C'est à Walter Wiora que l'on doit la caractérisation générale du jodel: «Dans la terminologie scientifique, "jodel" désigne un chant sans paroles, avec alternance permanente de registres. Contrairement à la norme d'égalisation des registres, la voix saute constamment du registre de "poitrine" à celui de "tête" (chez les hommes, voix de sifflet ou de fausset), allant de l'un à l'autre sans transition. Elle suit des degrés musicaux [...]. Normalement, on [...] jodle sur des [...] syllabes propres au jodel $»^{3}(1958: 73)$. Selon cette conception, il en résulterait un mouvement mélodique caractérisé par des intervalles disjoints et opposant deux timbres vocaux qui vont jusqu'à donner l'impression de plurivocalité. Il est généralement admis que le jodel se distingue de la simple brisure vocale par le fait que le chanteur opère dans un système scalaire défini avec des degrés bien déterminés.

La littérature relative à notre sujet témoigne d'une grande confusion terminologique en ce qui concerne la notion de registre. Les différentes disciplines auxquelles touche cette étude utilisent le terme de «registre» dans des sens extrêmement différents. La linguistique l'utilise avec des significations presque contradictoires: les africanistes déterminent ainsi les «niveaux» tonaux d'une langue à tons (Diki-Kidiri 1988: 2), alors que René Gsell, dans sa description de plusieurs langues à tons d'Asie du Sud-Est, appelle ainsi les qualités pertinentes de la voix qui s'ajoutent à la marque de distinction de «niveaux» de hauteurs tonales (Gsell 1979: 4). La musicologie s'y réfère soit pour désigner des timbres instrumentaux réalisés par l'orgue, soit pour l'attribuer aux niveaux mélodiques d'une polyphonie liés à des régions de hauteurs distinctes, ou encore aux différentes trames d'une monodie. Quant à la terminologie relative au chant qui nous intéresse plus spécialement ici, elle est loin d'être univoque. Dans la dernière, il y a notamment confusion entre le critère de hauteur du son et celui de mode vibratoire des cordes vocales comme paramètre déterminant d'un registre vocal (Laver 1980, Hollien 1971 et 1974). D'après les travaux de Michèle Castellengo, le tuilage des registres vocaux peut atteindre jusqu'à deux octaves. Par conséquent, la définition du registre qui se réfère à la fréquence fondamentale de la note chantée nous paraît insatisfaisante pour les besoins de notre analyse.

Par registre vocal, il faut donc comprendre ici l'ensemble de la qualité d'une voix, tributaire des conditions physiologiques de la production du son.

«Im wissenschaftlichen Sprachgebrauch bedeutet Jodeln textloses Singen mit fortwährendem Registerwechsel. Entgegen der Norm des Registerausgleichs springt hier die Stimme immer wieder vom "Brust"- in das "Kopf”-Register (bei Männern in Fistel oder Falsett) zurück, indem sie von dem einen zum andern unvermittelt umschlägt oder umbricht. Sie hält sich dabei an musikalische Tonstufen [...]. Gewöhnlich jodelt man [...] auf Jodelsilben ». 


\section{Les registres vocaux du jodel}

La «voix de poitrine» est le mode vibratoire par excellence de la parole et du chant simple. Dans les chants des Pygmées Aka, elle correspond à toute exécution non jodlée, qu'elle soit monodique ou polyphonique. Dans les parties jodlées, elle représente l'un des deux timbres constitutifs de la «polyphonie inhérente » à cette technique vocale.

La voix de poitrine couvre une grande étendue de fréquences moyennes, environ deux octaves. La vibration des cordes vocales est de grande amplitude, employant toute la masse musculaire. La consistance compacte des cordes vocales, alliée au fait que les fréquences émises dans ce mode vibratoire couvrent avant tout la tessiture inférieure de la voix, suscite une impression acoustique de densité. Celle-ci est confirmée par le nombre important d'harmoniques dans le spectre des sons dont le nombre exact change en fonction du volume du son et de la forme des cavités de résonance.

Le timbre opposé, dans le jodel, à celui émis par la voix de poitrine, est tributaire de la «voix de fausset». Elle couvre la tessiture supérieure de la voix sur une étendue moyenne d'une octave et demie. Ce sont uniquement les marges des cordes vocales, amincies et très tendues, qui vibrent librement en des mouvements peu complexes et le spectre est alors plus pauvre en harmoniques que celui produit par la voix de poitrine. Puisque les sons chantés en fausset sont généralement plus aigus, leurs harmoniques sont plus espacés en fréquences et moins intenses. Il en résulte une sensation de légèreté du son qui inspire des comparaisons avec celui d'une flûte.

La validité de ces caractéristiques pour le registre aigu du jodel n'est pas incontestée. En effet, Richard Luchsinger, après avoir travaillé avec des chanteurs autrichiens qui adaptaient à leur chant la technique du support du souffle, constate que la «voix de jodel» est plus riche en harmoniques que la voix de poitrine (1970: 224). La technique du support du souffle étant développée pour le chant classique occidental - en vue d'un mélange des registres - nous pouvons rapprocher la «voix de jodel» de ce que Luchsinger appelle «voix de tête» dans une étude consacrée au chant non jodlé. Elle se réfère à un son aigu masculin qui serait «un produit sonore [...] constitué également de résonance de poitrine. Par corollaire, il est plus riche en harmoniques que la voix de fausset ${ }^{4}(1947: 507)$. Devant ces résultats, F. Frank et M. Sparber insistent sur la distinction entre fausset et fausset soutenu (1972:165), alors que, selon la définition de Walter Graf, ces derniers seraient équivalents dans le jodel (1975: 609). Les différents concepts sont résumés dans le tableau ci-après:

4 «...ein Klangprodukt [...] dem aber auch Brustresonanz beigemischt ist. Demgemäss ist er viel reicher an Obertönen als das Falsett». 


\begin{tabular}{|l|l|c|c|c|}
\hline Auteur & Registre vocal & $\begin{array}{c}\text { Plus pauvre } \\
\text { que la voix } \\
\text { de poitrine }\end{array}$ & $\begin{array}{c}\text { Plus riche } \\
\text { que la voix } \\
\text { de fausset }\end{array}$ & $\begin{array}{c}\text { Plus riche } \\
\text { que la voix } \\
\text { de poitrine }\end{array}$ \\
\hline \multirow{2}{*}{ Luchsinger } & fausset & $\times$ & & \\
\cline { 2 - 6 } & voix de tête & & $\times$ & \\
\hline \multirow{2}{*}{ chant normal } \\
\hline \multirow{2}{*}{ Graf jodlé } & $\times$ & & \\
\hline \multirow{2}{*}{ Frank et Sparber } & fausset & & & \\
\cline { 2 - 6 } & fausset soutenu & & & \\
\hline Luchsinger & voix de jodel & & & \\
\hline
\end{tabular}

Tableau 1: Caractéristiques du registre aigu du jodel.

On constate qu'il n'y a pas de divergence sur le terme de fausset, mais que pour un même phénomène, celui du son plus riche que le fausset, les deux termes «fausset soutenu» et «voix de tête» sont utilisés selon qu'ils appartiennent au chant normal ou au jodel.

Nous supposons que des interdépendances entre mécanisme vibratoire et activité du diaphragme peuvent mener à un tel développement du «fausset soutenu» qu'il engendre plus d'harmoniques que la voix de poitrine. Dans ce cas, afin de ne garder qu'un seul terme par concept, nous proposons d'éliminer le terme «voix de jodel» et de lui réserver celui de «voix de tête».

Plus loin, nous étudierons la pertinence de la distinction entre «fausset», «fausset soutenu» et «voix de tête» pour la musique aka. Afin d'éviter de nouvelles confusions et en attendant une détermination du registre aigu du jodel pygmée, nous adopterons, bien qu'elle nous semble très rigide, la terminologie de Castellengo ${ }^{5}$ qui parle de «mécanisme I» et «mécanisme II» pour désigner les aspects physiologiques des registres vocaux impliqués dans le jodel, et de «registre grave» et «registre aigu» pour ce qui est des impressions acoustiques respectives qui s'en dégagent.

Les deux mécanismes se rejoignent en alternance pour former le tissu de timbres propre au jodel. L'instant qui les relie, la «brisure» proprement dite, en est un élément caractéristique, indépendamment de la civilisation qui l'utilise. Cette brisure est «cachée» dans le chant classique occidental, mais elle est parfaitement audible dans une voix non éduquée (Rubin et Hirt 1960: 1322). La transition tout à fait naturelle d'un mode vibratoire à l'autre est

\footnotetext{
Voir aussi l'article de Michèle Castellengo dans ce numéro des Cahiers.
} 
donc exploitée de façon à devenir partie intégrante de l'expression artistique qu'est le jodel.

Dans toutes les études sur le jodel, la caractéristique du changement des registres vocaux est toujours décrite comme étant étroitement liée à l'utilisation d'intervalles disjoints. La prédominance de ces intervalles est en effet propre à toute réalisation du jodel, y compris dans la musique aka. Néanmoins, vu le grand tuilage entre les deux mécanismes, il n'est pas impossible, comme on le verra plus loin, de s'imaginer un jodel incorporé dans une mélodie à intervalles et ambitus restreints.

\section{Les voyelles du jodel}

Essentiellement, le jodel a pour appui articulatoire des voyelles. Celles-ci sont souvent précédées de consonnes d'attaque qui facilitent le passage d'un registre à un autre.

A partir des informations dont nous disposons, nous regroupons dans le tableau suivant l'inventaire des voyelles utilisées dans le jodel, indépendamment de leur origine culturelle (tableau 2).

Ce tableau, bien qu'il embrasse des musiques de deux continents dont les cultures sont très différentes, se révèle cependant très cohérent. On y voit que dans le registre grave sont utilisées les voyelles $[e, \varepsilon, a, o, o]^{6}$ et dans le registre

\begin{tabular}{|l|l|l|l|}
\hline Région & Registre grave & Registre aigu & Auteur \\
\hline Autriche & ho, jo & di, ri & (Frank/Sparber) \\
\hline \multirow{2}{*}{ Alpes } & $\mathrm{o}$ & $\mathrm{i}$ & (Wiora) \\
\cline { 2 - 3 } & $\mathrm{o}$, o, a, $\varepsilon, \mathrm{e}$ & $\mathrm{i}, \mathrm{y}, \mathrm{u}$ & (Graf 1969) \\
\hline \multirow{2}{*}{ Afrique centrale } & $\mathrm{a}, \varepsilon$ & $\mathrm{i}$ & (Strasbaugh) \\
\cline { 2 - 3 } & $\mathrm{e}$ & $\mathrm{i}$ & (Pepper) \\
\hline \multirow{2}{*}{ Gabon } & $\mathrm{e}, \mathrm{a}$ & $\mathrm{i}$ & (Sallée) \\
\cline { 2 - 3 } & $\mathrm{a}, \mathrm{o}, \varepsilon$ & $\mathrm{i}, \mathrm{e}$ & (Carrington) \\
\hline \multirow{2}{*}{ Zaïre } & $\mathrm{ke}, \mathrm{le}$ & $\mathrm{ki}, \mathrm{li}$ & \\
\cline { 2 - 3 } & $\mathrm{ko}, \mathrm{o}$ & $\mathrm{ku}, \mathrm{u}$ & \\
\hline
\end{tabular}

Tableau 2: Répartition des voyelles sur les registres du jodel.

\footnotetext{
Toute voyelle figurant entre [ ] représente une notation phonétique.
} 
aigu les voyelles [i, y, u]. Les premières sont des voyelles ouvertes ${ }^{7}$, mi-ouvertes et mi-fermées alors que les secondes sont des voyelles fermées. La corrélation qui se dégage entre registre et voyelle est donc d'ordre physiologique: il y a correspondance entre le degré d'aperture et le registre. On remarque de plus que, à l'exception de l'exemple du Gabon, le registre aigu du jodel ne comprend que les voyelles du premier degré d'aperture $[\mathrm{i}, \mathrm{y}, \mathrm{u}]$. Les voyelles de tous les autres degrés d'aperture se trouvent dans le registre grave.

Afin de faciliter la compréhension d'une analyse sous son aspect acoustique, il convient d'être familiarisé avec quelques notions de phonétique acoustique. Chaque voyelle correspondant à une position bien définie des organes phonatoires, sa qualité sonore est déterminée par la forme spécifique du conduit vocal $^{8}$. Les cavités sont généralement présentées comme une série de résonateurs: l'onde glottale ${ }^{9}$, munie d'harmoniques, passe par les cavités qui renforcent les harmoniques correspondant à leur résonance propre (Sundberg 1981: 22). Ainsi se forment dans le spectre final des maxima d'énergie appelés les formants. La distribution d'énergie se fait en général sur plusieurs harmoniques voisins de la fréquence de résonance des cavités. Le tableau ci-dessous représente, comme illustration, les deux premiers formants de sept voyelles françaises:

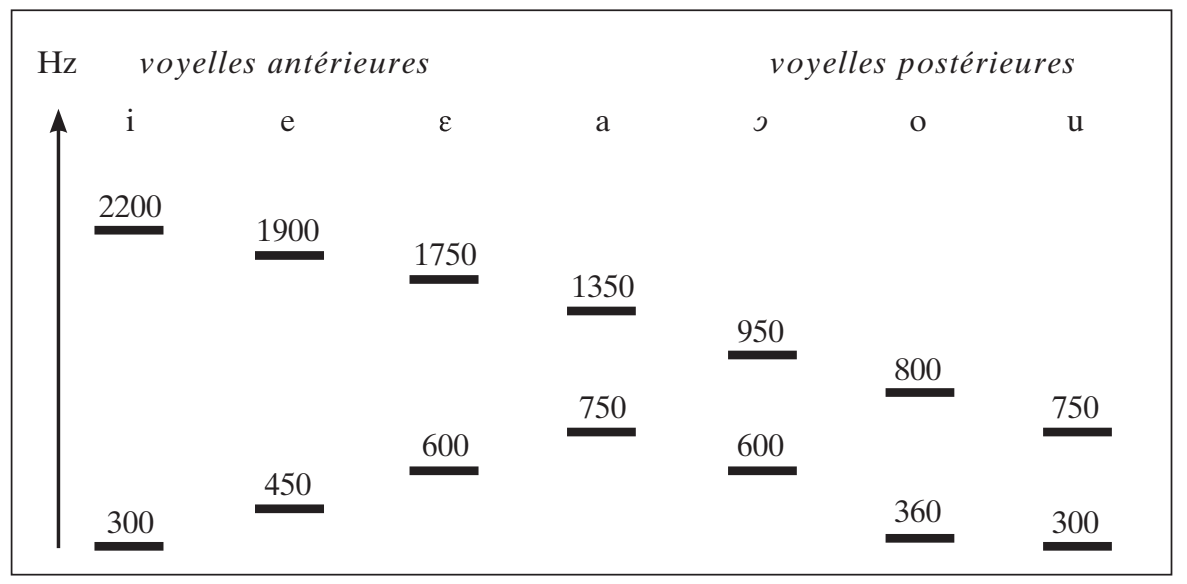

Tableau 3: Valeurs formantielles de quelques voyelles françaises.

Le trapèze vocalique classe les voyelles selon le point d'articulation de la langue et le degré d'aperture de la mâchoire:

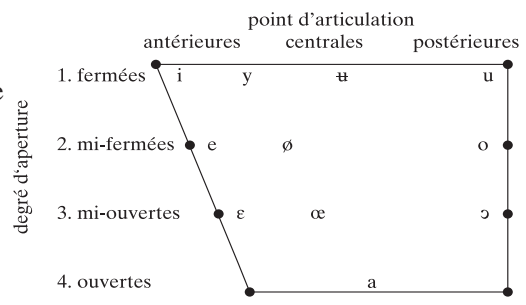

Le conduit vocal est constitué des cavités pharyngale, buccale, nasale et labiale.

$9 \quad$ L'onde vibratoire des cordes vocales représente le cycle d'ouverture et de fermeture de la glotte. 


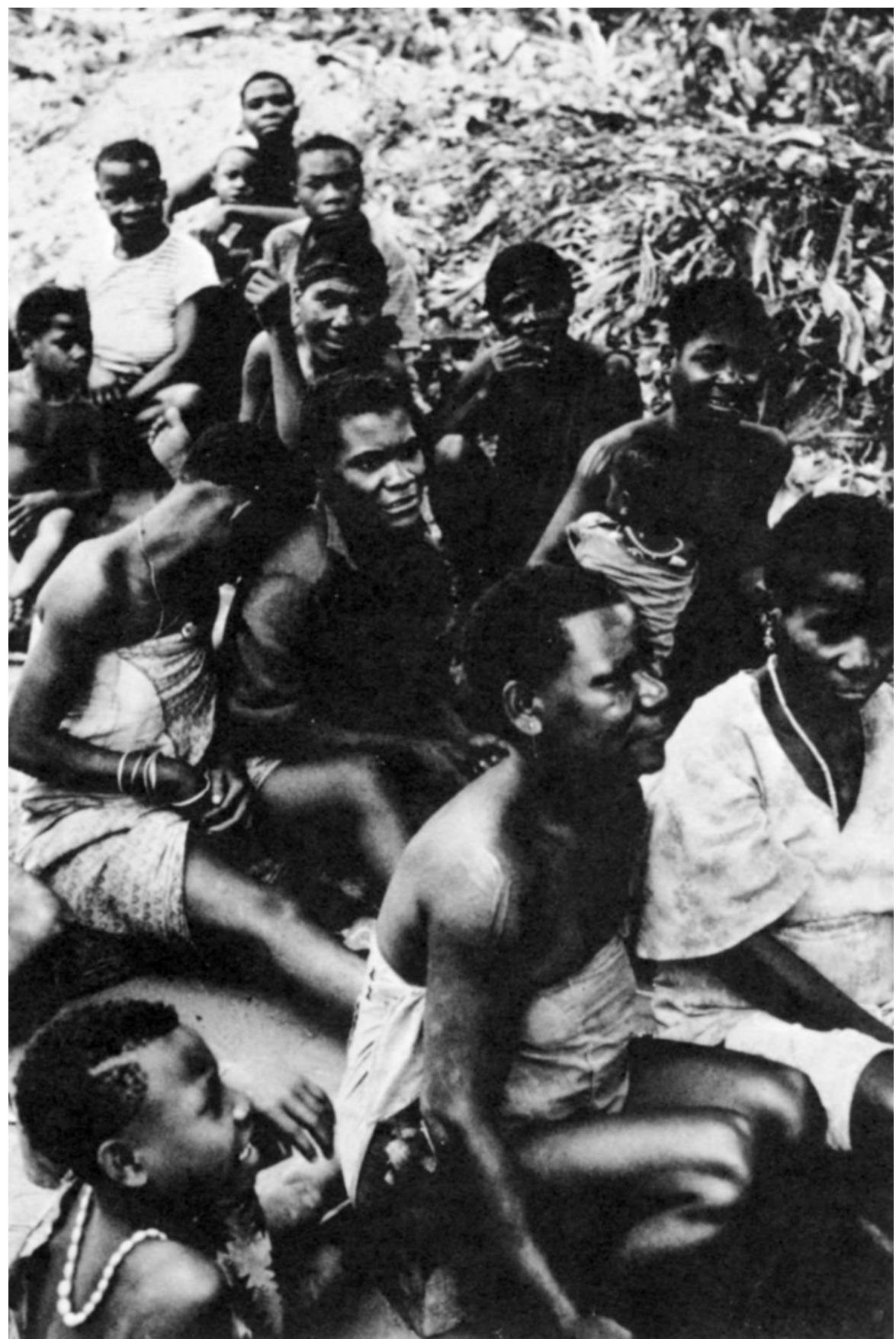

Enquête avec les chanteuses aka. Mongoumba, République Centrafricaine. Photo: Susanne Fürniß, 1989 


\section{Analyse de chants pygmées jodlés}

Nos analyses se basent sur des enregistrements de musique aka recueillis en République Centrafricaine par Simha Arom. Nous avons soumis à l'étude les différentes parties isolées d'un chant polyphonique qui nous serviront de référence pour quelques échantillons provenant d'autres pièces. Les analyses portent sur le chant díkóbódá mùdá sòmbé qui appartient à la catégorie des chants de divination bòndó. Comme toute polyphonie aka, il est constitué de quatre parties. Le mòtángòlè, chanté par un homme, est la voix principale qui contient les paroles du chant et sert de référence à toutes les autres voix. Le ngúe correspond à la voix basse, chantée par les hommes, et le òsêsê est une voix moyenne féminine. Comme le ngúé et le mòtángòlè, le òsêsê est chanté en mécanisme I. Le dìyèi, partie jodlée, est généralement chanté par les femmes et se superpose aux autres parties. La répartition selon les sexes n'est pas obligatoire puisque les trames des voix s'entrelacent dans la réalité; c'est pour cette raison qu'on trouvera également, dans les analyses qui suivent, des réalisations du dìyè par une voix d'homme. Dans le chant étudié, quelques parties existent en plusieurs versions. Des neuf voix du corpus, nous examinerons six parties: le mòtángòlè comme référence de mécanisme I et les cinq réalisations du dìyè chantées par deux personnes de sexe différent. Nous donnons ici un bref exemple de jodel transcrit:

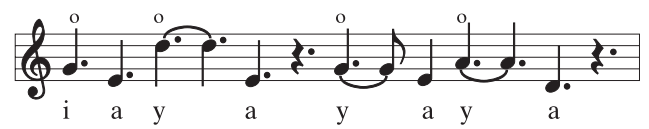

Les «o» sur les notes indiquent que celles-ci sont chantées en mécanisme II. Les transcriptions révèlent une alternance très régulière des deux modes vibratoires qui n'est que rarement interrompue par des notes de passage dans un même registre.

\section{Les voyelles}

Toutes les voyelles du jodel sont produites en dehors du contexte des paroles significatives. Pourtant, il est fort probable que se produisent des parallèles avec la langue parlée, les voyelles chantées étant produites avec le même appareil musculaire qui est entraîné à fonctionner selon les règles de la langue. Les sept voyelles de la langue aka sont /i, e, $\varepsilon$, a, ç, o, u/ ${ }^{10}$ (Cloarec-Heiss et Thomas 1978: 84-90). Dans le jodel, nous pouvons constater trois voyelles supplémentaires dont la plus fréquente est le [y]. En effet, cette dernière se trouve souvent sur des notes tenues ou à la note supérieure d'un intervalle de septième mineure. Dans la langue aka, le [y] existe comme variante phonétique du /u/. Jodlée, sa position est la même que celle du [u] ou du [i], elle conserve donc son caractère de variante pour une voyelle du premier degré d'aperture. A la même position se rencontre également une voyelle intermédiaire entre le [y] et

10 Toute voyelle figurant entre // représente une notation phonologique. 
le $[\mathrm{u}]$ que nous allons noter $[\mathrm{t}]$. Cette voyelle n'apparaît pas dans les variantes phonétiques de l'aka, ce qui est aussi le cas des voyelles $[\varnothing]$ et [œ]. Bien que rarement utilisées, elles figurent toujours dans le registre grave. Nous tendons alors à conclure que le jodel ne se sert pas uniquement des voyelles phonétiques d'une langue donnée, mais qu'en fonction du timbre vocal et du confort de réalisation de cette technique vocale, la musique s'impose à l'articulation jusqu'à créer ses propres voyelles.

Sur le corpus examiné, la répartition des voyelles sur les deux registres du jodel est la suivante:

registre aigu: $[\mathrm{i}] 46 \%-[\mathrm{u}] 26 \%-[\mathrm{y}] 20 \%-[\mathrm{t}] 8 \%$;

reg. grave: $[\varepsilon] 41 \%-[\mathrm{a}] 37 \%-[\mathrm{\rho}] 20 \%-[\propto] 1 \%-[\varnothing] 1 \%-[\mathrm{o}] 1 \%$.

Ce résultat coïncide avec les informations dont nous disposions antérieurement. Qui plus est, il renvoie à une distance vocalique très nette entre les deux registres du jodel. En effet, le registre aigu ne contient que des voyelles fermées et le registre grave des voyelles ouvertes et mi-ouvertes. A l'exception de quelques-unes $[o, \varnothing]$, les voyelles mi-fermées sont quasiment absentes et n'apparaissent que dans une ligne descendante, dans laquelle se succèdent exceptionnellement deux notes du registre grave:

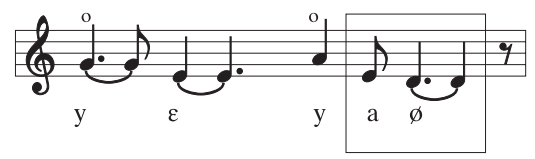

Ces cas semblent contredire le principe d'alternance des registres. Mais, en remplaçant une voyelle fermée - chantée normalement dans le registre aigu - par une voyelle du deuxième degré d'aperture, l'alternance de voyelles miouvertes/mi-fermées donne l'impression acoustique du jodel sans cependant changer de registre vocal. Ce phénomène permet de modifier la ligne mélodique tout en gardant le trait caractéristique du changement de timbre du jodel. On peut donc constater que les voyelles du deuxième degré d'aperture, en tant que voyelles-charnières, ont une fonction intermédiaire qui ne les intègre pas directement dans l'inventaire vocalique du jodel. Celui-ci est constitué de voyelles fermées, mi-ouvertes et ouvertes.

Puisque le registre aigu ne contient que des voyelles du premier degré d'aperture, les notes de passage ne peuvent pas jouer sur le degré d'aperture des voyelles. Elles semblent fonctionner selon un autre procédé illustré par l'exemple suivant:

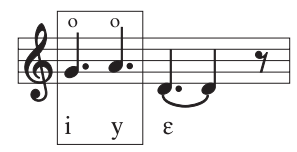

L'arrondissement des lèvres lors de la production du [y] rétrécit le passage du souffle par rapport à l'émission du [i] étiré, et le [y] pourrait être considéré comme une voyelle «très fermée» qui s'oppose aux voyelles de son entourage. 
L'inventaire vocalique du jodel démontre que l'espace articulatoire entre les [i] et les [u] se remplit avec les voyelles intermédiaires [y] et [u]. De plus, les valeurs des premiers formants révèlent un rapprochement de l'articulation des voyelles opposées. On peut donc constater qu'une économie articulatoire est due, dans le jodel, à la réalisation efficace de l'enchaînement des voyelles. Les spécificités acoustiques des registres du jodel aka peuvent être déterminées à partir de l'analyse de sonagrammes à bande étroite ${ }^{11}$. Nous avons extrait des cinq parties jodlées un échantillon de trente motifs contenus dans une unité de souffle et dont chacun comprend entre cinq et neuf notes correspondant à autant de voyelles. Les registres grave et aigu vont être examinés d'abord isolément, ensuite dans leur enchaînement en unités minimales jodlées.

\section{Mécanisme I - registre grave}

Comme le montre la figure suivante, le mécanisme I se caractérise par une série de composantes successives d'intensité considérable, atteignant au moins $4000 \mathrm{~Hz}$ :

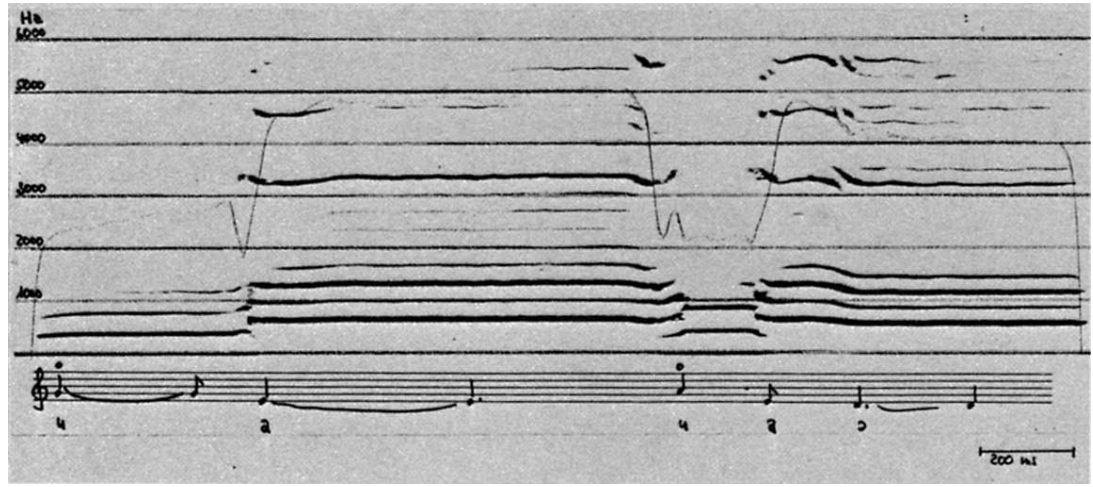

Sonagramme 1

L'incidence des voyelles sur le mécanisme I est importante du fait que les harmoniques se suivent de très près dans l'espace sonore. Celui-ci est couvert d'un dense filet de composantes, et les changements spectraux provoqués par l'enchaînement des voyelles sont facilement captés par les harmoniques. Ils sont, de ce fait, aisément perceptibles. Les harmoniques situés près des formants sont prédominants dans la composition spectrale. Cependant, ce mode de vibration étant puissant du fait de l'importance de la masse musculaire mise en vibration, les harmoniques localisés entre les zones formantielles sont également développés et le son reflète une densité perceptible. Puisque les voyelles

11 Les sonagrammes ont été effectués sur un appareil Voice Print 700 Series à l'Institut de phonétique de l'Université de Paris III - Sorbonne Nouvelle. Je remercie Zoubir Yacoubi de m'avoir initiée à la constitution et à l'interprétation des sonagrammes. 
du registre grave sont des voyelles ouvertes, d'un premier formant relativement élevé (voir tableau 3), la fréquence fondamentale est toujours inférieure à la valeur formantielle. Ceci a pour conséquence qu'elle est en général très faible ou absente sur le tracé ${ }^{12}$ :

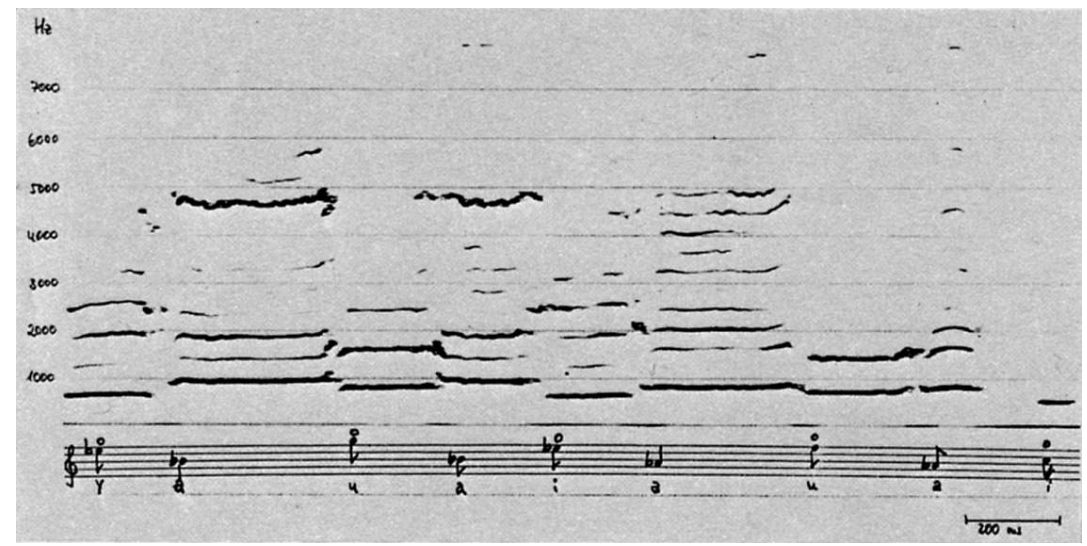

Sonagramme 2

Le sonagramme 2 représente un motif jodlé qui englobe entre autres quatre sons du registre grave, les sib et lab, chantés sur la voyelle [a]. Cet exemple illustre le phénomène de la fondamentale absente: les formants renforcent en effet le premier harmonique au-dessus de la fondamentale qui, elle, n'est pas visible. Il apparaît alors que le déplacement de l'énergie spectrale entre le mécanisme I et le mécanisme II se dirige, dans le jodel, dans un sens opposé à celui du mouvement mélodique. Dans le cas d'un renforcement très accentué des formants, l'impression acoustique peut même aller jusqu'à être perçue comme contraire au mouvement mélodique ${ }^{13}$.

Mises à part quelques exceptions peu nombreuses, les sons du registre grave se présentent de façon homogène.

\section{Mécanisme II - registre aigu}

Dans l'examen du registre aigu, nous allons essayer d'utiliser les termes «fausset», «fausset soutenu» et «voix de tête», termes mentionnés plus haut, afin de voir s'ils sont pertinents dans le cadre de la musique étudiée.

Si l'on considère les sons avec trois harmoniques comme étant des sons chantés en «fausset», nous en trouvons chez tous les chanteurs aka. Chez les

\footnotetext{
Les études sur les sons sans fondamentale ont montré que l'oreille est capable d'en déduire la hauteur correcte si elle dispose d'une série successive d'harmoniques (Risset 1978).

13 Je remercie Franz Födermayr de m'avoir communiqué cette observation faite par Werner Deutsch lors d'une audition publique.
} 
deux chanteurs de référence, de telles réalisations occupent $28,5 \%$ des sons du registre aigu alors que les deux autres chanteuses utilisent presque exclusivement le «fausset».

Ces sons, dont nous donnons deux exemples de sonagrammes, se trouvent dans la plupart des cas en position initiale dans une série d'environ cinq notes qui forment une unité de souffle:

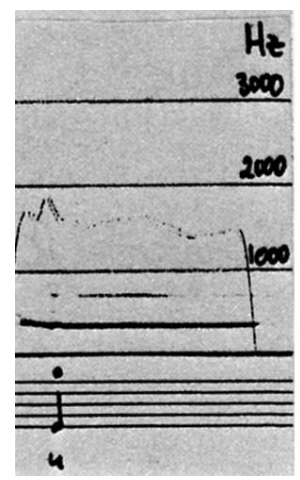

Sonagramme 3

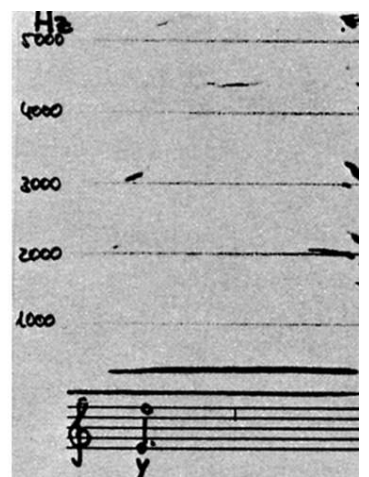

Sonagramme 4

Le sonagramme 3, représentant une note finale qui n'est jodlée que très rarement, montre deux harmoniques bien définis ainsi qu'un troisième, à peine visible, à $3000 \mathrm{~Hz}$. Le sonagramme 4, en revanche, laisse apparaître un seul harmonique d'intensité importante, les trois autres n'étant que partiellement visibles. Ceci nous servira pour démontrer comment le sonagraphe enregistre les sons selon la capacité d'audition de l'oreille humaine. Le spectre DFT ${ }^{14} \mathrm{du}$

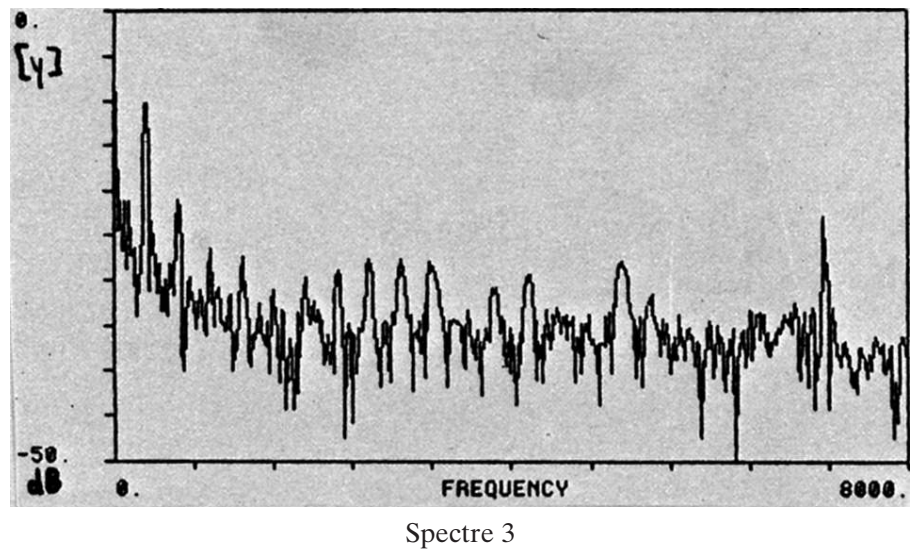

Discrete Fourier Transform. Ces analyses ont été faites au Laboratoire universit. d'application de la physique (LUAP) à l'Université de Paris VII - Jussieu sur une association d'un ordinateur PDP 11.04 à un Macintosh II. Je remercie Mokhtar Ghalala de m'avoir initiée à cette technique. 
troisième exemple, exposé ci-dessous, révèle une richesse sonore de dix harmoniques répartis jusqu'à $4500 \mathrm{~Hz}$, dont le premier, le troisième et le septième sont les seuls à apparaître sur le sonagramme.

Il faut noter que seules les composantes d'une intensité de $20 \mathrm{~dB}$ supérieure au bruit de fond sont enregistrées par le sonagraphe, et le tracé de l'exemple 3 ne se distingue pas essentiellement de celui d'un son véritablement pauvre comme il est représenté dans la figure suivante:

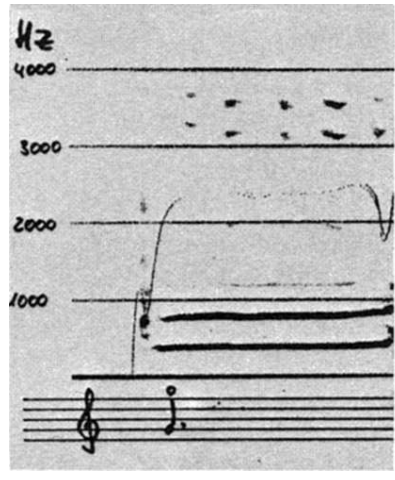

Sonagramme 5

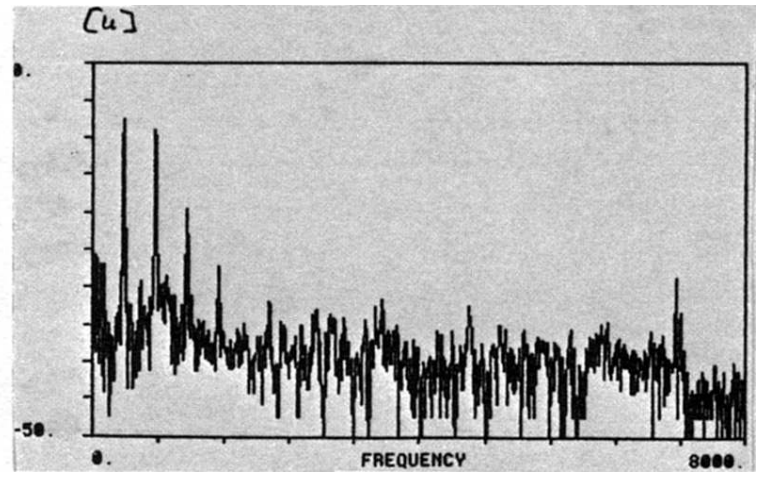

Spectre 6

Nous constatons donc l'importance éminente de l'amplitude des harmoniques, paramètre plus pertinent que le nombre des composantes présentes sur le sonagramme. Ainsi l'équation «fausset = pauvre»se relativise, la richesse potentielle qui gère la catégorisation n'étant pas visible sur le sonagramme.

En ce qui concerne les sons plus riches du registre aigu, il se dégage une tendance à les diviser arbitrairement en «fausset soutenu» et «voix de tête».

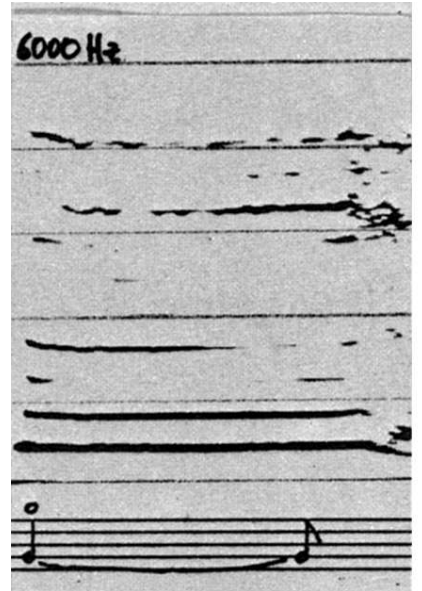

Sonagramme 6

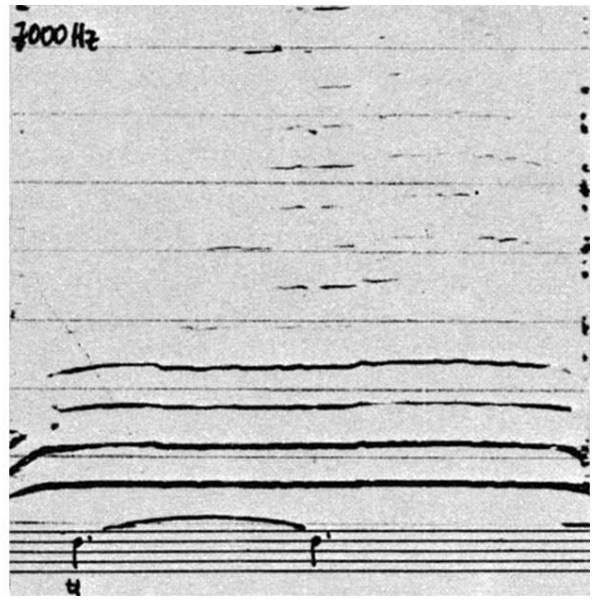

Sonagramme 7 
Le premier présente quatre à six harmoniques (sonagramme 6) qui ne sont pas successifs et dont le dernier correspond à peu près au huitième. La «voix de tête» (sonagramme 7) met en valeur un spectre plus homogène qui monte jusqu'au treizième harmonique. Par conséquent, il apparaît que le champ spectral de la «voix de tête» est plus large que celui du «fausset soutenu».

L'image du sonagramme 6 est très caractéristique des sons aigus du jodel pygmée. Il est rare, en effet, que les composantes visibles se succèdent, et leur évolution temporelle est instable. De plus, on trouve souvent un renforcement des harmoniques autour de $4000 \mathrm{~Hz}$.

La richesse de la voix de tête visible sur le sonagramme 7 est bien mise en valeur dans le spectre DFT:

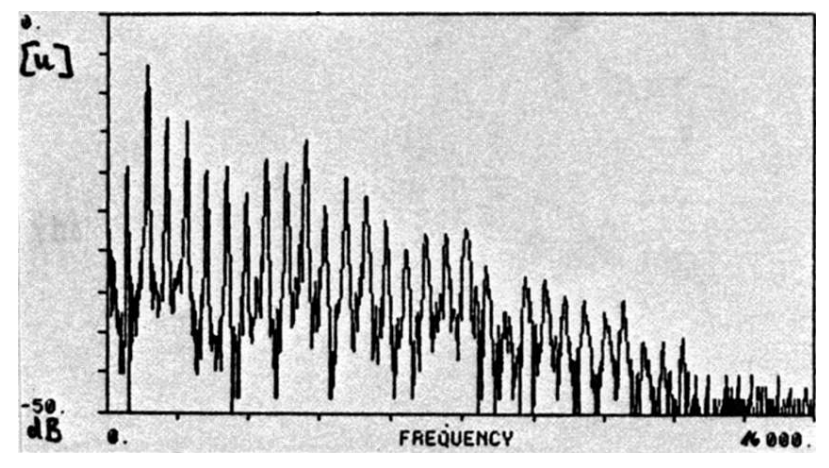

Spectre 7

Cet exemple est le seul où l'extension spectrale de la voix d'homme dépasse $4000 \mathrm{~Hz}$ avec une série successive et significative d'harmoniques, la femme en réalise plusieurs. Ce spectre, avec vingt-neuf composantes, monte jusqu'à $13000 \mathrm{~Hz}$ tout en gardant une intensité importante au-delà de $8000 \mathrm{~Hz}$.

Comment se présente la corrélation entre les voyelles utilisées et la constitution du registre aigu? La répartition des réalisations du mécanisme II en fonction des voyelles étudiées ne révèle pas d'interdépendance. En effet, les traits distinctifs des voyelles opposées, c'est-à-dire le point d'articulation ${ }^{15}$ pour les oppositions $[\mathrm{i}, \mathrm{y} / \mathrm{t}, \mathrm{u}]$ et la position des lèvres pour les oppositions $[\mathrm{i}, \mathrm{t} / \mathrm{y}, \mathrm{u}]$, ne permettent pas de regroupements des sons chantés. Les deux extrêmes - le [i] antérieur étiré et le [u] postérieur arrondi - réalisés par la chanteuse, se classent dans la même catégorie de «fausset», tandis que, plus riches dans les réalisations du chanteur, ils se trouvent dans les catégories de «fausset soutenu» et de voix de tête.

Il apparaît ainsi que le registre aigu du jodel pygmée témoigne d'une composition spectrale variée et que la plupart des sons comportent au moins de quatre à six harmoniques, parfois jusqu'à treize. L'intensité respective des composantes est faible, à l'exception de celles des zones formantielles. On observe souvent la

\footnotetext{
Voir note 7.
} 


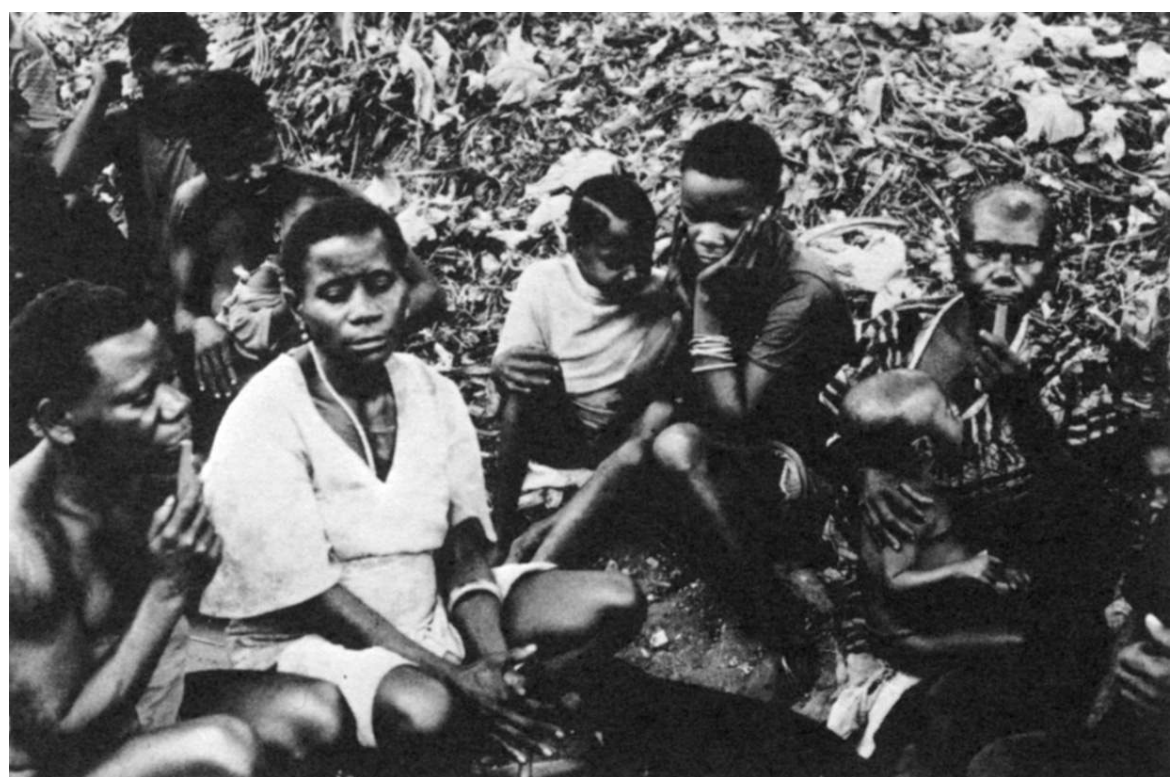

Des femmes aka jouant des sifflets. Mongoumba, Rép. Centrafricaine. Photo: Susanne Fürniss, 1989

présence de spectres pauvres en position initiale du motif chanté plutôt qu'en position intermédiaire, sans cependant exclure cette dernière possibilité.

En ce qui concerne la dénomination du registre aigu, une distinction entre «fausset», «fausset soutenu» et «voix de tête» ne nous paraît pas pertinente pour le jodel aka. Il n'y a pas de critères de délimitation valables qui se dégagent de l'analyse acoustique. Les caractéristiques font apparaître qu'il s'agit bien du mécanisme II, et cela paraît être une caractérisation suffisante dans le cas du jodel concerné.

\section{L'alternance des registres}

Selon la constitution spectrale des unités minimales jodlées, qui sont déterminées par un seul changement de mécanisme vibratoire, on peut diviser le corpus en trois catégories provisoires qui, pour les besoins de l'explication, seront nommées «pauvre», «riche» et «équilibré»:

1. jodel «pauvre»: le registre grave comporte plus d'harmoniques que le registre aigu et occupe un espace spectral plus large que celui-ci;

2. jodel «riche»: le registre aigu occupe un plus grand espace spectral et présente plus d'harmoniques que le registre grave;

3. jodel «équilibré»: les deux registres occupent le même espace dans le spectre. 
La première catégorie englobe des réalisations diverses:

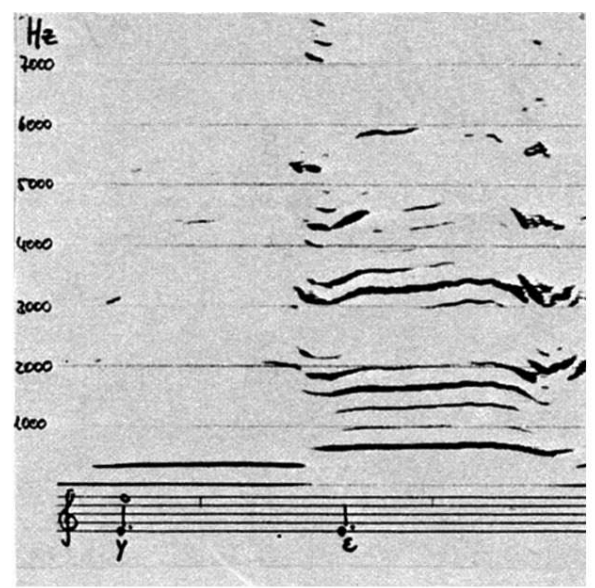

Sonagramme 8

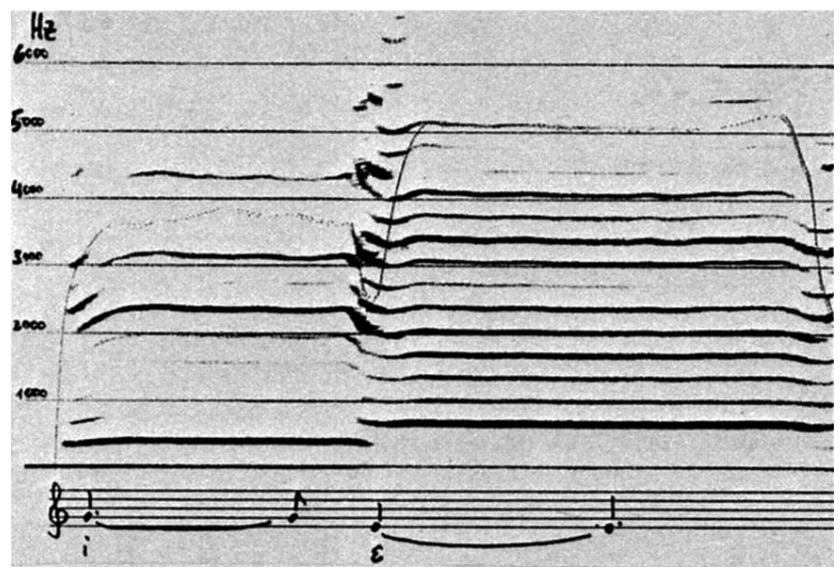

Sonagramme 9

Dans le cas du sonagramme 8, le contraste entre l'indigence du son aigu et la richesse en harmoniques du son grave est remarquable. Dans le sonagramme 9, cependant, le mécanisme II relativement riche se rapproche du mécanisme I, mais les qualités sonores restent distinctes du fait de la largeur spectrale occupée. Le nombre d'harmoniques présents et l'énergie sonore par secteur d'analyse de l'oreille restent inférieurs pour le mécanisme II et ceci donne au son sa légèreté qualitative. Le mécanisme I est caractérisé par une densité sonore résultant de la concentration de l'énergie dans un champ de fréquences restreint.

L'examen du «jodel riche» révèle que l'étendue du registre aigu monte audessus de $7000 \mathrm{~Hz}$ : 


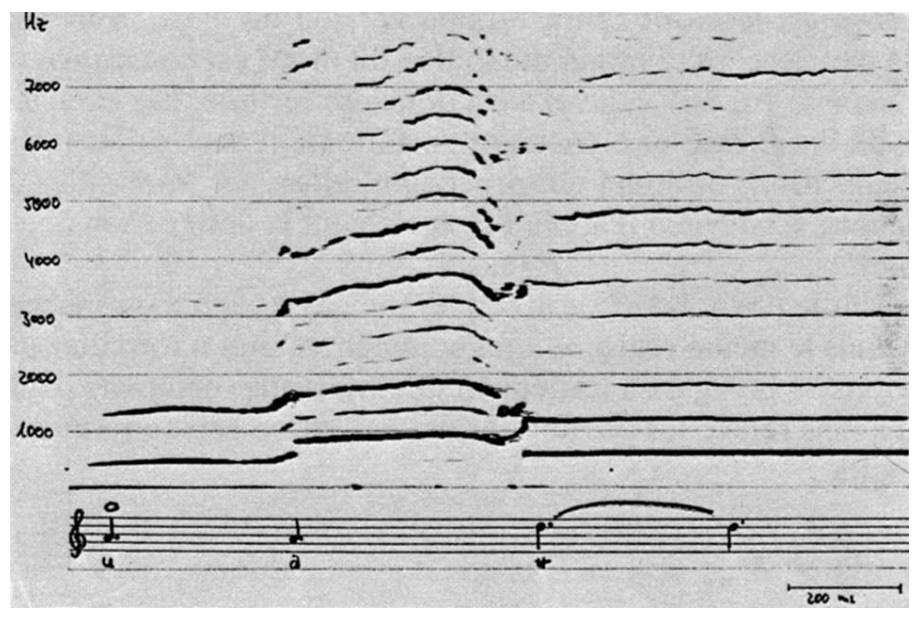

Sonagramme 10

Cet extrait illustre l'extraordinaire variété de l'expression jodlée chez les Pygmées: «fausset» et "voix de tête» se trouvent en voisinage immédiat. Les harmoniques $\mathrm{du}[\mathrm{t}]$ témoignent d'une grande régularité jusqu'à la limite $\mathrm{du}$ champ d'analyse vertical du sonagraphe.

Considérant les résultats de nos analyses, nous sommes amenés à constater de nouveau la non-pertinence d'une distinction entre «fausset «, «fausset soutenu» et «voix de tête» pour le jodel aka et nous nous contentons de définir le registre aigu comme étant tributaire du mécanisme II. Le sonagramme 10 touche encore à une des questions que nous tentons d'élucider dans cette étude: le jodel est-il lié à la réalisation de grands intervalles? Dans le cas observé, les deux premiers sons se trouvent sur le même degré de l'échelle du système tonal utilisé, bien qu'appartenant à deux registres différents. Les formants des voyelles se déplacent sur d'autres harmoniques. La fondamentale du [a] est à peine visible tandis que celle $\mathrm{du}[\mathrm{u}]$ est très bien développée. L’image montre en effet la brisure provoquée par le jodel: la densité sonore du [a] du registre grave, dépendant de l'intensité et de l'espace restreint entre les harmoniques, s'oppose à celle $\mathrm{du}[\mathrm{t}] \mathrm{du}$ registre aigu, beaucoup moins homogène et moins développée. Cet exemple pourrait servir d'illustration de ce qui est le timbre d'un son, c'est-à-dire la spécificité qui distingue deux sons de même durée et de hauteur et d'intensité identiques.

D'autres motifs témoignent également de la présence d'intervalles conjoints dans le jodel. Dans le système pentatonique utilisé par les Pygmées Aka, des tons distants d'une tierce mineure sont à considérer comme des degrés conjoints tout comme les secondes majeures:

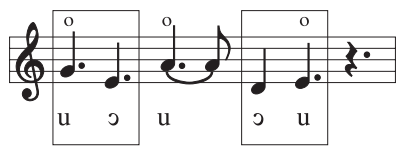


Cet extrait est le motif central d'une version du dìyèr. Tout au long de la pièce, la dernière unité minimale jodlée du motif est constituée d'une seconde ascendante (ré-mi); celle-ci n'est donc pas fortuite. Les caractéristiques spectrales de ces exemples coïncident parfaitement avec celles révélées par d'autres réalisations du jodel qui procèdent, elles, par intervalles disjoints. Par conséquent, il convient d'appliquer également la désignation de «jodel» à ces exemples.

Comparant le phénomène de secondes jodlées à celui d'une succession de deux sons dans le même registre, nous sommes amenés à formuler une hypothèse à propos de la règle du traitement des intervalles conjoints dans le jodel aka. Nous avons relevé les possibilités de réalisation suivantes au sein d'une unité de souffle:

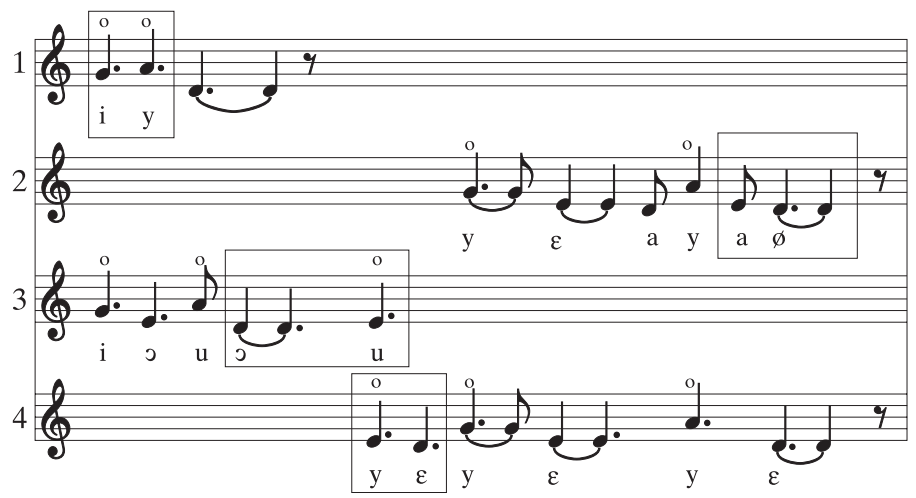

Dans la première ligne, deux sons en mécanisme II sont enchaînés dans un mouvement ascendant (sol-la), alors que dans la deuxième, une seconde descendante (mi-ré) est chantée en mécanisme I. Reliant ceci aux intervalles conjoints jodlés des lignes 3 et 4 (ré-mi et mi-ré respectivement), nous observons que la deuxième note d'un intervalle ascendant appartient toujours au registre aigu et celle d'un intervalle descendant au registre grave. Il se dégage alors une corrélation déterminée par le mode vibratoire de la première note de l'intervalle chanté. Nous constatons le principe suivant:

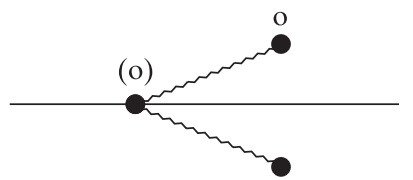

Principe du traitement des intervalles conjoints dans le jodel des Pygmées Aka.

Nous n'avons pas trouvé de réalisations de secondes ascendantes en mécanisme I ni de secondes descendantes en mécanisme II. L'élargissement du corpus à 23 parties jodlées d'autres chants polyphoniques a révélé que 97,5\% des 
intervalles conjoints correspondent à ce principe. Cela est tout à fait cohérent si l'on considère que le mode vibratoire qui représente le registre grave ne peut pas être utilisé au-dessus d'un son du registre aigu et vice versa. Le mode vibratoire de la première note de l'intervalle conjoint dépend de sa position dans la mélodie, elle-même étroitement liée au modèle mélodique et à ses variantes.

Jusqu'ici, on avait tendance à considérer que la caractéristique du jodel serait l'alternance de registres vocaux et leur association intime à l'emploi d'intervalles disjoints. Or, la musique des Pygmées Aka montre à l'évidence que ceci n'est pas la seule manière de jodler. Le jodel peut être produit sur des intervalles conjoints (seconde majeure, tierce mineure) tout autant que sur l'émission répétée d'une seule et même hauteur. Cela est confirmé par une analyse acoustique qui révèle que la sensation de «brisure» caractéristique du jodel a pour fondement l'alternance de deux densités différentes, indépendamment de leurs hauteurs respectives.

On constate que, quel que soit le contexte culturel dans lequel le jodel est effectué, la répartition des voyelles est systématique: le registre aigu est exécuté sur des voyelles du premier degré d'aperture, le registre grave sur celles des troisième et quatrième degrés. Quant aux voyelles du deuxième degré - et ceci paraît être une spécificité pygmée -, elles n'apparaissent que dans une succession de hauteurs au sein du registre grave pour y remplacer les voyelles fermées. Nous sommes alors conduits à proposer la définition suivante: Le jodel résulte de l'alternance des mécanismes I et II, chantés avec des voyelles spécifiques sur des degrés constitutifs d'un système scalaire.

Le mécanisme II est partie intégrante de la mélodie au même titre que le mécanisme I. Le chant prend appui sur des voyelles dont le degré d'aperture détermine leur appartenance à l'un ou l'autre des registres. Les voyelles fermées se trouvent systématiquement dans le registre aigu alors que les voyelles ouvertes se situent toujours dans le registre grave. Malgré la prédominance d'intervalles disjoints, on peut constater que, quel que soit l'intervalle qui sépare les deux éléments constitutifs d'une unité jodlée - y compris l'absence d'intervalle -, l'alternance des deux mécanismes vibratoires n'est pas nécessairement associée à une différentiation de hauteur. 


\section{Bibliographie}

BRĂILOIU Constantin

1949 «A propos du jodel», in: Kongressbericht der Internationalen Musikgesellschaft 1940 4: 6971.

CARRINGTON John F.

1971 «The musical dimension of perception in the Upper Congo, Zaïre». African Music 5, $1: 46-71$.

CLOAREC-HEISS France et THOMAS Jacqueline M.C.

1978 L'aka, langue bantoue des Pygmées de Mongoumba (Centrafrique). Paris: SELAF.

DIKI-KIDIRI Marcel

1987 «Accents et tons». Papier présenté au Séminaire de DEA du LACITO (CNRS).

FRANK F. et SPARBER M.

1972 «Neue klanganalytische Erkenntnisse über das Jodeln aus phoniatrisch-gesangspädagogischer Sicht». Folia phoniatrica (Basel, München) 24(3): 161-8.

FÜRNISS Susanne

s.d. DieJodeltechnik der Aka-Pygmäen in Zentralafrika. Eine akustisch-phonetische Untersuchung. Sprache und Oralität in Afrika. Berlin: Dietrich Reimer Verlag. (Sous presse).

s.d. «Falsetto con variazioni. Le fausset dans le monde sonore des Pygmées Aka (Centrafrique)». Annales d'Audiophonologie (à paraître).

GRAF Walter

1965 «Naturwissenschaftliche Gedanken über das Jodeln». Schriften des Vereins zur Verbreitung naturwissenschaftlicher Kenntnisse in Wien 105: 2-25.

1969 «Die musikalische Klangforschung: Wege zur Erfassung der musikalischen Bedeutung der Klangfarbe». Musik und Gesellschaft 6. Karlsruhe: G. Braun.

1975 «Sonagraphische Untersuchungen mit Beispielen aus dem deutschsprachigen Volksgesang», in: R.W. Brednich, L. Röhrig u. W. Suppan (eds.) Handbuch des Volksliedes, Vol. 2: 583622. München: Wilhelm Fink Verlag.

GSELL René

1979 «Études et recherches tonales (contribution à la typologie tonale)». Soutenance sur travaux. Exposé de synthèse. Paris: Institut de Phonétique.

HORNBOSTEL Erich-Maria von

1925 «Über die Entstehung des Jodelns», in: Kongressbericht über den musikwissenschaftlichen Kongress in Basel 1924 : 320-31. Leipzig: Breitkopf u. Härtel.

HOLLIEN Harry

1971 «Three major vocal registers: a proposal», in: 7th International Congress of Phonetic Sciences, Montreal : 320-31.

1974 «On vocal registers». Journal of Phonetics 2(2): 125-43.

LADEFOGED Peter

1975 A course in phonetics. New York/Chicago: Harcourt Brace Jovanovich Inc.

LAVER John

1984 The Phonetic Description of Voice Quality. Cambridge/London/New York: Cambridge University Press.

LEIPP Emile

1984 Acoustique et musique. Paris/New York/Barcelone/Milan: Masson. [1 ${ }^{\text {ère }}$ éd. 1971].

LUCHSINGER Richard

1947 «Falsett und Vollton der Kopfstimme». Archiv für Ohren-, Nasen- und Kehlkopfheilkunde 155: 505-19.

1970 «Die Jodelstimme», in: R. Luchsinger \& G.E. Arnold (eds.) Lehrbuch der Stimm- und Sprachheilkunde, Vol. 1: 222-26. Wien. 


\section{PEPPER Herbert}

s.d. Anthologie de la vie africaine: Moyen-Congo, Gabon. Notice du coffret de disque DucretetThomson 320C : 126-128.

RISSET Jean-Claude

1978 Hauteur et timbre des sons. Paris: Centre Georges Pompidou. [Rapport IRCAM 11].

RUBIN H.J. et HIRT Ch.C

1960 «The falsetto. A high cinematographic study». Laryngoscope 70: 1305-24.

\section{SALLÉE Pierre}

s.d. Pygmées et Bochimans. Notice de disque. Musiques et traditions du Monde CBS 80212.

STRASBAUGH Lamar Gene

1972-1973 «Two lullabies from the BaBinga BaBenzélé Pygmies - Transcriptions, analysis and commentary». Mitteilungen der deutschen Gesellschaft für Musik des Orients 11: 79-101.

\section{SUNDBERG Johan}

1981 «Le chant», in: Sons et musique: 20-7. Paris: Pour la Science. [Bibliothèque «Pour la Science»].

THOMAS Jacqueline M.C., BOUQUIAUX Luc et CLOAREC-HEISS France

1976 Initiation à la phonétique. Paris: PUF.

WINCKEL Fritz

1967 «Phoniatric acoustics», in: R. Luchsinger \& G.E. Arnold (eds.) Voice-Speech-Language. Clinical Communicology: its Physiology and Pathology: 24-55. Belmont.

WIORA Walter

1958 «odeln», in: F. Blume (ed.) Musik in Geschichte und Gegenwart Vol. 7: col. 74-79. Kassel/ Basel: Bärenreiter. 